\title{
Diagnosis of D-Bifunctional Protein Deficiency through Whole-Genome Sequencing: Implications for Cost-Effective Care
}

\author{
Alina Khromykh $^{a} \quad$ Benjamin D. Solomon ${ }^{a}$, b, d Dale L. Bodian ${ }^{a}$ Eyby L. Leon ${ }^{f}$ \\ Ramaswamy K. lyer $^{a, e}$ Robin L. Baker ${ }^{b, c}$ David P. Ascher ${ }^{b, d}$ Rajiv Bavejab, c \\ Joseph G. Vockley ${ }^{a, c}$ John E. Niederhuber ${ }^{a}$ \\ a Inova Translational Medicine Institute, ${ }^{b}$ Inova Children's Hospital, Inova Health System, and 'Fairfax Neonatal \\ Associates, Falls Church, Va., Departments of ${ }^{\mathrm{d}}$ Pediatrics and ${ }^{\mathrm{O}}$ Obstetrics and Gynecology, Virginia Commonwealth \\ University School of Medicine, Richmond, Va., and 'Division of Genetics and Metabolism, Children's National \\ Medical Center, Washington, D.C., USA
}

\author{
Key Words \\ Clinical workup - Cost-effective care · D-Bifunctional protein \\ deficiency . Whole-genome sequencing
}

\begin{abstract}
D-Bifunctional protein deficiency, caused by recessive mutations in HSD17B4, is a severe disorder of peroxisomal fatty acid oxidation. Nonspecific clinical features may contribute to diagnostic challenges. We describe a newborn female with infantile-onset seizures and nonspecific mild dysmorphisms who underwent extensive genetic workup that resulted in the detection of a novel homozygous mutation (c.302+1_4delGTGA) in the HSD17B4 gene, consistent with a diagnosis of $D$-bifunctional protein deficiency. By comparing the standard clinical workup to diagnostic analysis performed through research-based whole-genome sequencing (WGS), which independently identified the causative mutation, we demonstrated the ability of genomic sequencing to serve as a timely and cost-effective diagnostic tool for the molecular diagnosis of apparent and occult newborn diseases. As genomic sequencing becomes more available and af-
\end{abstract}

(C) 2015 S. Karger AG, Base

$1661-8769 / 15 / 0063-0141 \$ 39.50 / 0$ fordable, we anticipate that WGS and related omics technologies will eventually replace the traditional tiered approach to newborn diagnostic workup.

(c) 2015 S. Karger AG, Basel

\section{KARGER 125}

E-Mail karger@karger.com www.karger.com/msy
Advances in whole-exome sequencing (WES) and whole-genome sequencing (WGS) technologies have increasingly generated opportunities for efficient detection of disease-causing mutations and structural variants in a diverse group of medical conditions. For many disorders of the newborn, the resulting molecular diagnosis may offer definitive information that can help direct patient care [Solomon et al., 2013, 2015].

In clinical genetics, as the cost of WES and WGS continue to decrease and analysis becomes more efficient and accurate, these technologies are becoming the standard to solve the difficult-to-diagnose inherited diseases of the newborn [Roach et al., 2010; Saunders et al., 2012; Biesecker and Green, 2014].

We describe here the use of research-based WGS in an infant whose presentation involved neonatal hypotonia 
and early-onset seizures. Our individual research analyses are bolstered by our database of $>6,000$ clinically annotated WGS derived from multiple ongoing trio-based genomic sequencing studies at our research institute [Solomon, 2014].

WGS enabled us to identify a homozygous deletion in the HSD17B4 gene, leading to a diagnosis of D-bifunctional protein deficiency in this infant. The application of WGS in this case is presented together with a comparison to the standard clinical testing timeline using available diagnostics. The results illustrate the potential of WGS to resolve diagnostic quandaries for patients in a timely and cost-effective manner. We conclude that the WGS approach will eventually replace the relatively expensive and slow 'shotgun' or 'tiered' approach to diagnostic testing [Soden et al., 2014].

\section{Materials and Methods}

The study 'The Incidence and Burden of Congenital Anomalies, Genetic Disorders and Genetic Suspicion in Neonatal and Pediatric Patients' was approved by the Western Institutional Review Board, with full informed consent obtained from all participants. In this research protocol, we seek to evaluate the genetic and genomic causes of undiagnosed pediatric diseases using WGS and related genomic technologies. Our study cohort is accessioned in a single, large (75 isolettes, with additional flex space), level IV neonatal intensive care unit (NICU) as well as from the pediatric intensive care unit, which are both part of the larger Inova Children's Hospital. In addition to the presence of a possible genetic/genomic but molecularly undiagnosed disorder, further inclusion/exclusion criteria for the research study are summarized as follows: the proband must be the biological offspring of the parents (cases of nonparentage ascertained through WGS are 'silently' dropped from analysis); parents must be at least 18 years of age; there must be no parental history of cancer in the last 5 years, so that mutations that may be related to cancer or treatment will not affect analyses, and both parents must be willing to enroll in the research study to allow trio-based WGS and related investigations. Other relatives may also participate and typically undergo WGS concurrently with the primary trio [Bodian et al., 2014; Solomon et al., 2015].

We collect one set of samples from the family, including blood and saliva from each family member. Samples from parents are collected at their convenience by a trained staff member at the bedside or in our clinical or research phlebotomy units. Proband samples are coordinated with the standard clinical workup so as not to impose additional burdens or risks on the newborn and care team. Additional family members may also contribute samples.

Clinical information is captured through a single electronic medical record system. This information is supplemented with questionnaires and longitudinal surveys administered every 6 months in order to gather information that is not part of the electronic medical record, such as diet and nutrition, potential environmental exposures, country of ancestral origin, and detailed demographic data. Additional medical findings are identified through the electronic medical records for participants for whom a molecular or other etiology remains unidentified in order to search for further diagnostic clues that may be identified after the initial NICU hospitalization.

For further details on methods, see our publications describing WGS to elucidate the causes of congenital disorders [Bodian et al., 2014; Stittrich et al., 2014; Solomon et al., 2015].

\section{Sample Collection and Processing}

Whole blood is collected from participants in BD vacutainers (for parents) or microtainers (for infants) potassium (K2) EDTA tubes. Genomic DNA (gDNA) is isolated using a Qiagen DNAeasy kit on a QiaSymphony DNA isolation platform. DNA quality control and quantification are performed by spectrophotometry on a NanoDrop 800 instrument, and fluorimetry using Quantifluor dye on a TECAN F200 instrument.

WGS is performed using Illumina Inc's FastTrack WGS service, which generates high-quality WGS data, at a minimum of $30 \times$ average coverage. All protocols were performed using kits and following the recommendations of the manufacturers. Paired-end libraries are manually generated from $500 \mathrm{ng}$ to $1 \mu \mathrm{g}$ of gDNA using the Illumina TruSeq DNA Sample Preparation Kit (Catalog No.: FC-121-2001). Library quality is assessed by gel electrophoresis, or chip electrophoresis on the Agilent BioAnalyzer, using the Agilent DNA 1000 chip. Libraries are quantified by qPCR as described in the Illumina Sequencing Library qPCR Quantification protocol. DNA libraries are denatured, diluted, and clustered onto v3 flow cells, using the Illumina TruSeq Cluster Kit v3 and the Illumina $\mathrm{cBot}^{\mathrm{TM}}$ system. Clustered v3 flow cells are then loaded onto HiSeq 2000 instruments and sequenced on 100-bp paired-end, nonindexed runs, using Illumina TruSeq SBS v3 Reagents. Illumina HiSeq Control Software and Real-Time Analysis are used on HiSeq 2000 sequencing runs for real-time image analysis and base calling.

After WGS, raw data and processed files are loaded onto secure hard drives and uploaded to a private, secure Amazon cloud instance. Further WGS data analysis as described below is performed in the cloud environment.

\section{WGS Analysis and Variant Filtering}

After WGS, read alignment to human reference assembly hg19 [Lander et al., 2001] and variant calling are conducted by the Illumina Whole Human Genome Sequencing Service Informatics Pipeline version 2.01-02 using iSAAC [Raczy et al., 2013]. Trio genomes are merged into a single VCF file with gVCFtools (https:// sites.google.com/site/gvcftools/), and variant filtering is performed with KGGSeq v0.3 [Li et al., 2012] with additional annotations through ANNOVAR [Wang et al., 2010]. Variant filters include: genotype quality $\geq 10$, read depth $\geq 4$, and fraction of reads with alternative allele $>25 \%$ at heterozygous sites and $>70 \%$ at homozygous sites. As causative mutations are expected to be rare, variants with a minor allele frequency $>0.3 \%$ in the 1000 Genomes Project and NHLBI GO Exome Sequencing Project (http://evs. gs.washington.edu/EVS/) data are excluded [The 1000 Genomes Project Consortium et al., 2012]. In the first stage of analysis, variants are limited to SNPs and small insertions/deletions, with protein-affecting alleles (stop-gain, splice-site, and frameshift), and variants annotated as pathogenic in ClinVar VCF version12/30/13 or HGMD Professional version 2013.2 (Biobase) are prioritized [Landrum et al., 2014]. Genotype zygosity in the trio is required to 
be compatible with the phenotype pattern. Phenotypes resulting from known mutations in the genes containing the candidate variants are individually considered for compatibility with phenotypic manifestations.

\section{Variant Confirmation and Return of Results}

Identified variants of interest are confirmed via traditional bidirectional Sanger sequencing using the original research samples; results were CLIA validated separately (see below).

\section{Results}

\section{Clinical Description}

The female proband was born at 38 weeks of gestation via repeat $\mathrm{C}$-section due to cephalopelvic disproportion to a now 30-year-old G2P2 mother after an otherwise unremarkable pregnancy. The proband was the second child of a family of Middle Eastern descent; they have a reportedly healthy son. The parents denied knowledge of consanguinity. Genomic analysis also did not reveal areas of homozygosity that would suggest consanguinity.

Apgar scores were 8 and 9 at 1 and $5 \mathrm{~min}$, respectively. Immediately after birth, the child received routine care in the newborn nursery but was noted to have hypotonia, an inability to maintain adequate body temperature, and poor feeding (struggling to suck); the infant was transferred to the NICU at $\sim 6 \mathrm{~h}$ of life for further evaluation.

On admission to the NICU, the child was noted by the clinical genetics service to have several nonspecific mild dysmorphisms, including upslanting palpebral fissures, thick nasolabial folds with anteverted nares, mild retrognathia, excess nuchal skin, a wide intermammillary distance, and a wide space between the first and second toes. Overall, the physical examination was also felt to be consistent with reduced fetal movement, though this had not been noted during gestation by parental report or through standard prenatal care.

On the second day of life, the neonate developed clinically recognizable tonic-clonic seizures, and anticonvul-

Fig. 1. Clinical timeline demonstrating significant events, including clinical testing. See text for further details. Laboratory tests were performed on blood unless otherwise noted. Lines next to a test indicate the chronology of that test. As described, the availability of a single genomic test at initial concern for a disorder may frequently supplant a step-wise approach. $\mathrm{CMV}=$ Cytomegalovirus; $\mathrm{CSF}=$ cerebrospinal fluid; HSV = herpes simplex virus; TORCH = included toxoplasma IgM, rapid plasma reagin screening for syphilis, rubella IgM, CMV IgM, HSV 1, 2; UGI = upper gastrointestinal.

D-Bifunctional WGS

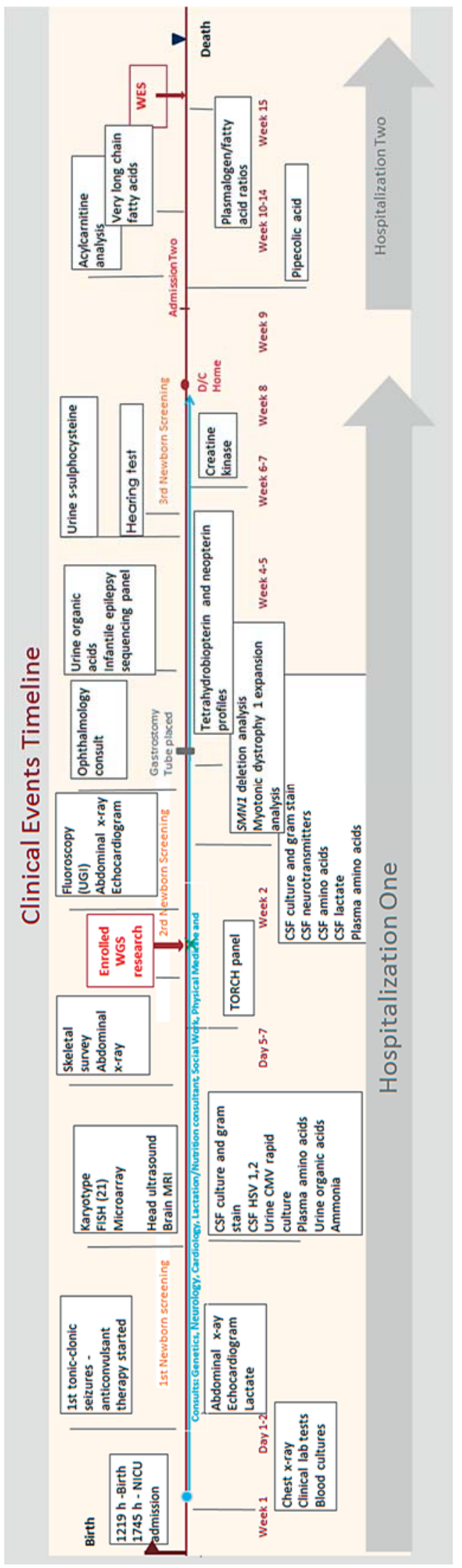

Mol Syndromol 2015;6:141-146 DOI: $10.1159 / 000433621$ 
sant therapy was initiated, including phenobarbital and levetiracetam. Several EEG studies were performed, which showed normal electrical activity of the brain in the awake and asleep states with no focal lateralizing or epileptiform pattern.

Brain MRI and cranial ultrasound were negative for acute processes or major structural neuroanatomical anomalies, although small bilateral subependymal cysts close to the foramen of Monro were noted. Ongoing feeding difficulties were partially addressed with gastrostomy tube insertion on the third week of life.

\section{Clinical Etiological Workup}

During the NICU hospitalization, the infant underwent a number of tests in an attempt to understand the cause of the child's condition (for a timeline of clinical events and testing, see fig. 1). Tests included TORCH testing (rapid plasma reagin screening for syphilis, rubella IgM, toxoplasma IgM, cytomegalovirus IgM, and herpes simplex virus testing performed via cerebrospinal fluid PCR) as well as blood and cerebrospinal fluid studies with culture to assess for infections. Testing for an inborn error of metabolism included: cerebrospinal fluid for neurotransmitters, amino acids, and lactate; plasma for amino acids and lactate, urine organic acids, and S-sulfocysteine assay, none of which were consistent with an inborn error of metabolism or other disorder.

Commercially performed cytogenetic or molecular testing on peripheral blood included: initial FISH for Trisomy 21, karyotyping, high-density microarray (oligonucleotide-single nucleotide polymorphism microarray via Affymetrix CytoScan $\mathrm{HD}$ ), spinal muscular atrophy via SMN1 deletion testing, myotonic dystrophy type 1 via repeat expansion analysis, and a panel of 38 genes involved in infantile epilepsy analyzed through both next-generation sequencing and deletion/duplication analysis. The epilepsy sequencing panel showed heterozygosity for a missense variant in the STXBP1 gene classified as a variant of unknown significance. All other results were negative.

Additionally, newborn screening (USA, Commonwealth of Virginia) was within normal limits. The brainstem auditory-evoked response test at 5 weeks of life was consistent with mild-to-moderate sensorineural hearing loss. Ophthalmic evaluation in the third week of life revealed no anomalies. A multidisciplinary team of clinicians (from the fields of neurology, cardiology, nutrition, infectious disease, and physical therapy and the clinical genetics team) provided consultations in order to help manage and identify the cause of the child's condition.
In parallel to standard clinical care, the family was offered participation in the WGS research study described above and enrolled when the infant was 11 days old. Six weeks after NICU admission, the child was discharged home, continuing anticonvulsant therapy and receiving nutrition via gastrostomy tube.

Due to the ongoing health concerns, seizures, and failure to thrive, the proband was readmitted to another area hospital and had additional diagnostic testing (this was concurrent with research-based WGS at the birth hospital). Among the tests performed at the outside hospital, the neonate was found to have a normal acylcarnitine profile and normal pipecolic acid, but she had an abnormal test result for very-long-chain fatty acids, consistent with a peroxisomal disorder.

For the research-based WGS analysis, variants passing the filtering criteria and consistent with de novo, homozygous recessive, and compound heterozygous inheritance models were evaluated manually for congruity of the encompassing genes with the observed phenotype. Examination of the 6 candidate de novo variants, 13 candidate homozygous recessive variants, and 56 candidate compound heterozygous variants in 21 genes led to a single homozygous variant in a gene with known function in accordance with the disease phenotype: a novel mutation (c.302+1_4delGTGA) in the HSD17B4 gene, consistent with D-bifunctional protein deficiency. Commercial WES through another medical center simultaneously documented this mutation. No proven treatments exist to address the underlying biochemical defect in this disorder, although some interventions are under investigation. In spite of the efforts of the medical team and the family, the child passed away at $\sim 6$ months of life.

\section{Discussion}

The power of genomic sequencing to achieve diagnoses of congenital disorders has been described in both small and relatively large studies and is being used in a number of institutions to identify disease-causing mutations for relevant patients [Yang et al., 2013; Biesecker and Green, 2014; Eng et al., 2014]. The cost of generating WGS data has decreased to USD 1,000 per sample, yet there are still valid concerns about the high cost of analysis and the time frame often required to achieve answers [Mardis, 2010; Hayden, 2014; Soden et al., 2014]. These issues are being addressed with new analytic methods and techniques that minimize the amount of time necessary to generate and analyze data. In fact, current methods 
point to the possibility that generation and clinically oriented analysis of WGS data may be available in the same time frame as a biochemical workup [Saunders et al., 2012; Stranneheim et al., 2014].

For the patient described here, for whom separate genomic sequencing modalities (WES and WGS, the latter of which has the advantage of being able to detect structural rearrangements as well as to interrogate nonexonic regions, though this was not necessary in this particular case) arrived at the same diagnosis, lending support for these approaches as a potential means of reducing the time, effort, and cost associated with achieving a molecular explanation for the disease [Soden et al., 2014]. If WGS were conducted immediately on suspicion of a genetic disorder, similar to the approach by which microarrays are often ordered in clinical practice, an answer would conceivably be available faster than through a tiered approach involving multiple rounds of testing, often suggested by multiple subspecialty consultants [Saunders et al., 2012; Korf and Rehm, 2013]. While there is controversy about the way that WGS and related modalities will affect the practice of clinical genetics, it is anticipated that the role of the clinical geneticist will increasingly shift to post hoc clinical correlations based on bioinformatics analyses of WGS data rather than choosing which individual molecular tests should be ordered [Hennekam and Biesecker, 2012; Solomon, 2014].

One counterargument specific to this case is that a more rapid and comprehensive biochemical workup, with targeted sequencing following the results, might have revealed the patient's etiology without necessitating genomic sequencing. Still, our clinically oriented research group, as well as others, frequently encounters situations such as this, where patients are not diagnosed through traditional means as rapidly as would be optimal. This may be either because a condition is extremely rare or because a presentation is atypical. Frequently, however, the lack of a rapid diagnosis through targeted testing may be due to the fact that every possibility is not covered by the relatively large number of studies that include all biochemical, cytogenetic, and molecular possibilities. In fact, we would posit that one advantage of genomic sequencing is that it is, in some ways, less operator dependent than the traditional workup process. This is especially apparent in regions of the world where there are fewer available genetic professionals who have been trained to recognize rare disorders. In other words, performing a single test (genomic sequencing) at the time of first clinical concern would have removed the possibility that another, narrow test would not be overlooked. This is even more relevant when one considers the fact that WGS can yield results in the same time frame as newborn screening or other biochemical studies [Stranneheim et al., 2014].

In the case described here, the cost of trio-based WGS was lower than the standard diagnostic workup performed at the birth hospital (see fig. 1). The USD 18,000 cost of the clinical diagnostic tests at the birth hospital alone was calculated based on actual reimbursements to the hospital. This estimation excludes the cost of WES, USD 7,000, which was performed outside the birth hospital. Early genomic sequencing may thus be highly cost-effective compared to the traditional cost of diagnostic testing, especially considering the costs for longer hospitalization, additional inpatient and outpatient physician consultations in an attempt to find the cause of the clinical presentation, other modalities that are used to help narrow the differential diagnosis (e.g. certain radiologic studies), medications that may be trialed, and other related services as well as the risk of iatrogenic complications from the diagnostic process [Soden et al., 2014]. Though the molecular explanation for this patient unfortunately does not yield a new management modality [Solomon et al., 2013], the knowledge may be beneficial in helping the family understand the cause of the condition as well as in informing their future reproductive decisions.

In conclusion, we illustrated the ability of WGS to act as a diagnostic tool. As genomic modalities such as WGS consistently achieve diagnoses with greater speed and lower cost, we anticipate that the use of WGS as an initial newborn test will become increasingly adopted as best practice [Saunders et al., 2012; Talkowski et al., 2012; Soden et al., 2014]. Health care systems, payors, and involved insurance reimbursement policies will eventually recognize that these technologies, when indicated, should be available to health care providers and their patients [Solomon, 2015].

\section{Acknowledgments}

This work was funded by the Inova Health System and a generous gift from the Odeen family. The authors would like to express their gratitude to the family for their participation in the study. 


\section{References}

Biesecker LG, Green RC: Diagnostic clinical genome and exome sequencing. N Engl J Med 370:2418-2425 (2014).

Bodian DL, Solomon BD, Khromykh A, Thach DC, Iyer RK, et al: Diagnosis of an imprintedgene syndrome by a novel bioinformtics analysis of whole-genome sequences from a family trio. Mol Genet Genomic Med 2:530-538 (2014).

-Eng CM, Yang Y, Plon SE: Genetic diagnosis through whole-exome sequencing. $\mathrm{N}$ Engl J Med 370:1068 (2014).

Hayden EC: Technology: The \$1,000 genome. Nature 507:294-295 (2014).

-Hennekam RC, Biesecker LG: Next-generation sequencing demands next-generation phenotyping. Hum Mutat 33:884-886 (2012).

Korf BR, Rehm HL: New approaches to molecular diagnosis. JAMA 309:1511-1521 (2013).

-Lander ES, Linton LM, Birren B, Nusbaum C, Zody MC, et al: Initial sequencing and analysis of the human genome. Nature 409:860921 (2001).

-Landrum MJ, Lee JM, Riley GR, Jang W, Rubinstein WS, et al: ClinVar: public archive of relationships among sequence variation and human phenotype. Nucleic Acids Res 42:D980-D985 (2014).

-Li MX, Gui HS, Kwan JS, Bao SY, Sham PC: A comprehensive framework for prioritizing variants in exome sequencing studies of Mendelian diseases. Nucleic Acids Res 40:e53 (2012).
Mardis ER: The $\$ 1,000$ genome, the $\$ 100,000$ analysis? Genome Med 2:84 (2010).

-Raczy C, Petrovski R, Saunders CT, Chorny I, Kruglyak S, et al: Isaac: ultra-fast whole-genome secondary analysis on Illumina sequencing platforms. Bioinformatics 29:20412043 (2013).

Roach JC, Glusman G, Smit AF, Huff CD, Hubley $\mathrm{R}$, et al: Analysis of genetic inheritance in a family quartet by whole-genome sequencing. Science 328:636-639 (2010).

$>$ Saunders CJ, Miller NA, Soden SE, Dinwiddie DL, Noll A, et al: Rapid whole-genome sequencing for genetic disease diagnosis in neonatal intensive care units. Sci Transl Med 4: 154ra135 (2012).

-Soden SE, Saunders CJ, Willig LK, Farrow EG, Smith LD, et al: Effectiveness of exome and genome sequencing guided by acuity of illness for diagnosis of neurodevelopmental disorders. Sci Transl Med 6:265ra168 (2014).

Solomon BD: Obstacles and opportunities for the future of genomic medicine. Mol Genet Genomic Med 2:205-209 (2014).

Solomon BD: Genomic sequencing and the impact of molecular diagnosis on patient care. Mol Syndromol 6:4-6 (2015).

$\checkmark$ Solomon BD, Nguyen AD, Bear KA, Wolfsberg TG: Clinical genomic database. Proc Natl Acad Sci USA 110:9851-9855 (2013).
Solomon BD, Bodian DL, Khromykh A, Mora GG, Lanpher BC, et al: Expanding the phenotypic spectrum in EP300-related RubinsteinTaybi syndrome. Am J Med Genet A 167: 1111-1116 (2015).

Stittrich AB, Lehman A, Bodian DL, Ashworth J, Zong Z, et al: Mutations in NOTCH1 cause Adams-Oliver syndrome. Am J Hum Genet 95:275-284 (2014)

Stranneheim H, Engvall M, Naess K, Lesko N, Larsson P, et al: Rapid pulsed whole genome sequencing for comprehensive acute diagnostics of inborn errors of metabolism. BMC Genomics 15:1090 (2014).

Talkowski ME, Ordulu Z, Pillalamarri V, Benson $\mathrm{CB}$, Blumenthal I, et al: Clinical diagnosis by whole-genome sequencing of a prenatal sample. N Engl J Med 367:2226-2232 (2012).

The 1000 Genomes Project Consortium; Abecasis GR, Auton A, Brooks LD, DePristo MA, et al: An integrated map of genetic variation from 1,092 human genomes. Nature 491:56-65 (2012).

-Wang K, Li M, Hakonarson H: ANNOVAR: functional annotation of genetic variants from high-throughput sequencing data. $\mathrm{Nu}$ cleic Acids Res 38:e164 (2010).

Yang Y, Muzny DM, Reid JG, Bainbridge MN, Willis A, et al: Clinical whole-exome sequencing for the diagnosis of Mendelian disorders. N Engl J Med 369:1502-1511 (2013). 